\title{
Local Tb Theorem on Spaces of Homogeneous Type
}

\author{
Chaoqiang Tan and Lixin Yan
}

\begin{abstract}
In this article, we obtain a local $\mathrm{Tb}$ theorem for singular integral operators on spaces of homogeneous type by using tree selection algorithm of the dyadic model and the BCR algorithm, which extends an earlier result of M. Christ [Colloq. Math. 60/61 (1990), 601-628].
\end{abstract}

Keywords. Local Tb theorem, singular integral operators, trees, BCR algorithm, spaces of homogeneous type

Mathematics Subject Classification (2000). Primary 42B20, secondary 42C40, 47B38

\section{Introduction}

We begin by recalling the definitions necessary for introducing singular integral theory on spaces of homogeneous type. A quasi-metric $\rho$ on a set $X$ is a function from $X \times X$ to $[0, \infty)$ satisfying

(i) $\rho(x, y)=0$ if and only if $x=y$;

(ii) $\rho(x, y)=\rho(y, x)$ for all $x, y \in X$;

(iii) there exists a constant $A<\infty$ such that for all $x, y$ and $z \in X, \rho(x, y) \leq$ $A(\rho(x, z)+\rho(z, y))$.

Any quasi-metric $\rho$ defines a topology, for which the balls $B(x, r)=\{y \in X$ : $\rho(y, x)<r\}$ for all $x \in X$ and $r>0$ form a basis.

Definition 1.1 ( [5]). A space of homogeneous type $(X, \rho, \mu)$ is a set $X$ together with a quasi-metric $\rho$ and a non-negative Borel measure $\mu$ on $X$ for which all the associated balls $B(x, r)$ satisfy the doubling property

$$
\mu(B(x, 2 r)) \leq C \mu(B(x, r))<\infty
$$

for all $x \in X$ and $r>0$, where the constant $C \geq 1$ is independent of $x$ and $r$. It is also required that $\mu(B(x, r))<\infty$ for all $x, r$.

C. Q. Tan: Department of Mathematics, Shantou University, Shantou Guangdong, 515063, P.R. China; cqtan@stu.edu.cn

L. X. Yan: Department of Mathematics, Zhongshan University, Guangzhou, 510275, P.R. China; mcsylx@mail.sysu.edu.cn 
In this paper, we will suppose that $\mu(X)=\infty$ and $\mu(\{x\})=0$ for all $x \in X$. For any $x, y \in X$, we set $\lambda(x, y)=\mu(B(x, \rho(x, y)))$. It is easy to see that $\lambda(x, y)$ is comparable to $\lambda(y, x)$, uniformly in $x, y$.

Definition 1.2. A standard kernel is a function $K(x, y): X \times X \backslash\{x=y\} \rightarrow \mathbb{C}$ such that there exist $\epsilon>0$ and $C<\infty$ such that

$$
|K(x, y)| \leq \frac{C}{\lambda(x, y)} \quad \text { for all distinct } x, y \in X
$$

and such that

$$
\left|K(x, y)-K\left(x^{\prime}, y\right)\right|+\left|K(y, x)-K\left(y, x^{\prime}\right)\right| \leq C\left(\frac{\rho\left(x, x^{\prime}\right)}{\rho(x, y)}\right)^{\epsilon} \frac{1}{\lambda(x, y)}
$$

whenever $\rho\left(x, x^{\prime}\right) \leq \frac{1}{2} \rho(x, y)$.

Denote by $\mathcal{D}_{\alpha}$ the space of all Hölder continuous functions of order $\alpha \in(0,1]$ with compact support and $\mathcal{D}_{\alpha}^{\prime}$ the dual space of $\mathcal{D}_{\alpha}$ (see [4, p. 603], for instance). We say that $T$ is a singular integral operator, if $T$ is a mapping from $\mathcal{D}_{\alpha}$ into $\mathcal{D}_{\alpha}^{\prime}$, which is associated to a standard kernel $K(x, y)$, in the sense that

$$
\langle T f, g\rangle=\iint K(x, y) f(y) g(x) d \mu(x) d \mu(y),
$$

where $f, g \in \mathcal{D}_{\alpha}$ with disjoint supports.

The first local Tb Theorem was proved by M. Christ [4]. It extends a global version due to David-Journé-Semmes [7] in that it permits a pseudo-accretive system, rather than a single para-accretive function.

Theorem 1.3 ( [4]). Suppose that $T$ is a singular integral operator associated to a standard kernel $K(x, y)$, which in addition we assume to be in $L^{\infty}$. Suppose also that there exist pseudo-accretive systems $\left\{b_{B}^{1}\right\},\left\{b_{B}^{2}\right\}$ such that $b_{B}^{1}$ and $b_{B}^{2}$ are supported in $B$, and for all ball $B$,

$$
\begin{aligned}
\left\|b_{B}^{1}\right\|_{L^{\infty}(B)}+\left\|b_{B}^{2}\right\|_{L^{\infty}(B)} & \leq C \\
\left\|T\left(b_{B}^{1}\right)\right\|_{L^{\infty}(B)}+\left\|T^{*}\left(b_{B}^{2}\right)\right\|_{L^{\infty}(B)} & \leq C \\
C^{-1} \mu(B) & \leq \min \left(\left|\int_{B} b_{B}^{1} d \mu\right|,\left|\int_{B} b_{B}^{2} d \mu\right|\right) .
\end{aligned}
$$

Then $T$ extends to a bounded operator on $L^{2}(X)$, with bounds independent of $\|K\|_{\infty}$.

It should be noted that the assumption that $K \in L^{\infty}$ is merely qualitative, and is satisfied, e.g., by smooth truncations of a standard kernel. This assumption allows one to make certain formal manipulations with impunity, during the course of the proof. See [4, p. 606]. 
In [1], P. Auscher et al. gave a generalization of a local Tb Theorem (of M. Christ) on $\mathbb{R}$. It is of perfect dyadic Calderón-Zygmund operators where the $L^{\infty}$ conditions are replaced by $L^{2}$ conditions. Perfect dyadic means essentially that the regularity is adapted to the dyadic grid: any function supported in a dyadic cube with mean 0 is mapped to a function supported in the same cube. Recently, P. Auscher and Q. X. Yang improved it for standard singular integrals where the $L^{2}$ conditions on the accretive system are replaced by the $L^{p}$ conditions for $1<p<\infty([2])$. The crucial idea is to write a standard singular integral as the sum of an $L^{p}$-bounded operator and of a dyadic perfect operator ([1]), where we can use the Beylkin-Coifman-Rokhlin algorithm (see $[2,8,10]$ ) to obtain the desired results.

The aim of this article is to generalize a local $\mathrm{Tb}$ theorem of [2] on $\mathbb{R}^{n}$ to general spaces of homogeneous type. The following is our main result.

Theorem 1.4. Suppose that $T$ is a singular integral operator associated to a singular kernel $K(x, y)$, which in addition we assume to be in $L^{\infty}$. Let $1<$ $p, q \leq \infty$ such that $\frac{1}{p}+\frac{1}{q} \leq 1$ with dual exponent $p^{\prime}, q^{\prime}$, i.e., $\frac{1}{p}+\frac{1}{p^{\prime}}=1$ and $\frac{1}{q}+\frac{1}{q^{\prime}}=1$. Suppose also that there exist pseudo-accretive systems $\left\{b_{B}^{1}\right\},\left\{b_{B}^{2}\right\}$ such that $b_{B}^{1}$ and $b_{B}^{2}$ are supported in $B$, and for all ball $B$,

$$
\begin{aligned}
\int_{B}\left|b_{B}^{1}\right|^{p} d \mu+\int_{B}\left|b_{B}^{2}\right|^{q} d \mu & \leq C \mu(B) \\
\int_{B}\left|T\left(b_{B}^{1}\right)\right|^{q^{\prime}} d \mu+\int_{B}\left|T\left(b_{B}^{2}\right)\right|^{p^{\prime}} d \mu & \leq C \mu(B) \\
C^{-1} \mu(B) & \leq \min \left(\left|\int_{B} b_{B}^{1} d \mu\right|,\left|\int_{B} b_{B}^{2} d \mu\right|\right) .
\end{aligned}
$$

Then $T$ extends to a bounded operator on $L^{2}(X)$, with bounds independent of $\|K\|_{\infty}$.

The paper is organized as follows. In Section 2, we introduce the notion of tree, martingale difference operator and dyadic BMO space on spaces of homogeneous type. In Section 3, we will give some estimates for singular integrals. The proof of our main result, Theorem 1.4, will be given in Section 4 by making use of the the dyadic model in [1] and the BCR algorithm in [2].

Throughout the paper, we use $A \lesssim B$ to denote the estimate $A \leq C B$ for some absolute constant $C$ which may vary from line to line.

\section{Notation and preliminaries}

We first state a result of M. Christ ( [4, Theorem 11]), which gives an analogue of the Euclidean dyadic cubes. 
Lemma 2.1. There exist a collection of open subsets $\mathbf{P}^{+}=\left\{Q_{k}^{j} \subset X: j \in\right.$ $\left.\mathbb{Z}, k \in I_{j}\right\}$, where $I_{j}$ denotes some (possible finite) index set depending on $j$, and constants $\delta \in(1, \infty), a_{0}>0, \eta>0$ and $C_{1}, C_{2}<\infty$ such that:

(i) $\mu\left(\left\{X \backslash \cup_{k} Q_{k}^{j}\right\}\right)=0$ for all $j \in \mathbb{Z}$.

(ii) If $j<j^{\prime}$, then either $Q_{k}^{j} \subset Q_{\ell}^{j^{\prime}}$, or $Q_{k}^{j} \cap Q_{\ell}^{j^{\prime}}=\emptyset$.

(iii) For each $(j, k)$ and each $j<j^{\prime}$ there is a unique $\ell$ such that $Q_{k}^{j} \subset Q_{\ell}^{j^{\prime}}$.

(iv) Diameter $Q_{k}^{j} \leq C_{1} \delta^{j}$.

(v) Each $Q_{k}^{j}$ contains some ball $B\left(z_{k}^{j}, a_{0} \delta^{j}\right)$.

(vi) $\mu\left\{x \mid \in Q_{k}^{j}: \rho\left(x, X \backslash Q_{k}^{j}\right) \leq C_{2} t \delta^{j}\right\} \leq C_{2} t^{\eta} \mu\left(Q_{k}^{j}\right)$ for any $k, j$ and $t>0$.

From the property (vi) of Lemma 2.1, it can be verified that for every $k, j$ and every $0<t \leq 1, \mu\left\{x \mid \in X \backslash Q_{k}^{j}: \rho\left(x, Q_{k}^{j}\right)\right\} \leq C_{2} t^{\eta} \mu\left(Q_{k}^{j}\right)$, and estimate which will be used in the sequel.

We call these open sets $\mathbf{P}^{+}$of Lemma 2.1 as being dyadic cubes. Whenever $Q_{k}^{j} \subset Q_{k^{\prime}}^{j+1}$, we shall say that $Q_{k}^{j}$ is a child of $Q_{k^{\prime}}^{j+1}$, and $Q_{k^{\prime}}^{j+1}$ the parent of $Q_{k}^{j}$. For every dyadic cube $Q$, the notation $\widetilde{Q}$ denotes the collection of all children of $Q$.

2.1. Trees. We follow an idea of [1] to describe a notion of tree on spaces of homogeneous type. A tree is a collection $T \subseteq \mathbf{P}^{+}$with a top dyadic cube $Q_{T} \in T$ such that $P \subseteq Q_{T}$ for all $P \in T$. If $P \in \mathbf{P}^{+}$, we define the complete tree $\operatorname{Tree}(P)$ to be the tree

$$
\operatorname{Tree}(P):=\left\{Q \in \mathbf{P}^{+}: Q \subseteq P\right\}
$$

with top $P$. If $T$ is a tree such that $T \subseteq \mathbf{P} \subseteq \mathbf{P}^{+}$, then we say that $T$ is complete with respect to $\mathbf{P}$ if $T=\operatorname{Tree}\left(Q_{T}\right) \cap \mathbf{P}$. For every $\alpha>0$, an $\alpha$-packing of $T$ is a set $\mathbf{P} \subseteq T$ such that $\sum_{P \in \mathbf{P}} \mu(P) \leq \alpha \mu\left(Q_{T}\right)$. We say that $\mathbf{P} \subseteq \mathbf{P}^{+}$is convex if for every pair of dyadic cubes $Q_{1} \subseteq Q_{2}$ in $\mathbf{P}$, the set $\left\{Q \in \mathbf{P}^{+}: Q_{1} \subseteq Q \subseteq Q_{2}\right\}$ is contained in $\mathbf{P}$. Consider a convex tree $T$ and a function $a: T \mapsto \mathbb{R}^{+}$, we define the size of $a$ on $T$ by

$$
\|a\|_{\operatorname{size}(T)}:=\frac{1}{\mu\left(Q_{T}\right)} \sum_{P \in T} a(P),
$$

and the maximal size of $a$ on $\mathbf{P}$ by

$$
\|a\|_{\text {size }^{*}(\mathbf{P})}:=\sup _{T \subseteq \mathbf{P}}\|a\|_{\operatorname{size}(T)},
$$

where $T$ ranges over all convex trees in $\mathbf{P}$. We adopt the convention that $\|a\|_{\text {size }^{*}(\mathbf{P})}=0$ if $\mathbf{P}$ is empty. Given any function $f$ on $X$ and a dyadic cube $Q \in \mathbf{P}^{+}$, we define

$$
\|a\|_{\operatorname{mean}(Q)}:=\mu(Q)^{-1} \int_{Q}|f| d \mu
$$


and for any collection $\mathbf{P} \subseteq \mathbf{P}^{+}$we define

$$
\|f\|_{\text {mean*(P) }}:=\sup _{Q \in \mathbf{P}}\|f\|_{\operatorname{mean}(Q)} .
$$

2.2. Martingle difference operators. For $1 \leq p \leq \infty$, the space of $p$ integrable functions on $X$ is denoted by $L^{p}(X)$, the norm of the function $f \in$ $L^{p}(X)$ by $\|f\|_{p}$, the scalar product in $L^{2}(X)$ by $\langle.,$.$\rangle , and [f]_{Q}=\mu(Q)^{-1} \int_{Q} f d \mu$.

For every $j \in \mathbb{Z}$, recall that $I_{j}$ denotes an index set depending on $j$ as in Lemma 2.1. Let $Q \in \mathbf{P}^{+}$. For every $f \in L^{2}(X)$, we define
a) $E_{Q}(f):=\mu(Q)^{-1} \int_{Q} f d \mu \cdot \chi_{Q}$
b) $\Delta_{Q}(f):=\sum_{Q^{\prime} \in \widetilde{Q}} E_{Q^{\prime}}(f)-E_{Q}(f)$
c) $\quad E_{j}(f):=\sum_{k \in I_{j}} E_{Q_{k}^{j}}(f)$
d) $\quad \Delta_{j} f:=\sum_{k \in I_{j}} \Delta_{Q_{k}^{j}}(f)$.

It can be verified that $\left\langle\Delta_{Q} f, \Delta_{Q^{\prime}} f\right\rangle=0$ whenever $Q \neq Q^{\prime}$. Then the following result holds. We omit the proof.

Lemma 2.2. For any $f \in L^{2}(X)$, we have the representation formula

$$
f=\sum_{Q \in \boldsymbol{P}^{+}} \Delta_{Q} f=\sum_{j \in \mathbb{Z}} \Delta_{j} f
$$

with $\|f\|_{2}^{2}=\sum_{Q \in \boldsymbol{P}^{+}}\left\|\Delta_{Q} f\right\|_{2}^{2}$.

2.3. Dyadic BMO space. If $T$ is a tree, we set $\Pi_{T} f:=\sum_{Q \in T} \Delta_{Q} f$, and hence $\Pi_{\operatorname{Tree}(Q)} f=\left(f-[f]_{Q}\right) \chi_{Q}$. Using the property of $\Delta_{Q} f$, we can estimate

$$
\left\|\Pi_{T} f\right\|_{2}^{2} \leq \sum_{Q \in \operatorname{Tree}\left(Q_{T}\right)}\left\|\Delta_{Q} f\right\|_{2}^{2} \lesssim \int_{Q_{T}}|f|^{2} d \mu .
$$

Definition 2.3. A locally integrable function $f$ defined on $X$ is said to be in the dyadic BMO space if $\|f\|_{\text {DBMO }}=\sup _{T} \mu\left(Q_{T}\right)^{-\frac{1}{2}}\left\|\Pi_{T} f\right\|_{2}<\infty$, where $T$ ranges over all trees belong to $\mathbf{P}^{+}$.

Lemma 2.4. Let $\mathbf{P} \subseteq \mathbf{P}^{+}$. Suppose that a is a function from $\mathbf{P}$ to $\mathbb{R}^{+}$. Then for any $1<p<\infty$,

$$
\sum_{Q \in \boldsymbol{P}} a(Q)\left|[f]_{Q}\right|^{p} \lesssim\|a\|_{s i z e^{*}(\mathbf{P})}\|f\|_{p}^{p}
$$


As a consequence, for every $f, g \in L^{2}(X)$ and every $h \in \mathrm{DBMO}$, we have that

$$
\sum_{Q \in \mathbf{P}^{+}}\left|\left\langle\Delta_{Q} f, h\right\rangle[g]_{Q}\right| \lesssim\|f\|_{2}\|g\|_{2}\|h\|_{\text {DBMO }}
$$

Proof. The proof of (10) is similar to that of [1, Theorem 5.1]. We omit it here. To prove (11), we use Hölder's inequality and (10) to obtain

$$
\begin{aligned}
\sum_{Q \in \mathbf{P}^{+}}\left|\left\langle\Delta_{Q} f, h\right\rangle[g]_{Q}\right| & \lesssim \sum_{Q \in \mathbf{P}^{+}}\left\|\Delta_{Q} f\right\|_{2}\left\|\Delta_{Q} h\right\|_{2}\left|[g]_{Q}\right| \\
& \lesssim\left\{\sum_{Q \in \mathbf{P}^{+}}\left\|\Delta_{Q} f\right\|_{2}^{2}\right\}^{\frac{1}{2}}\left\{\sum_{Q \in \mathbf{P}^{+}}\left\|\Delta_{Q} h\right\|_{2}^{2}\left|[g]_{Q}\right|^{2}\right\}^{\frac{1}{2}} \\
& \lesssim\|f\|_{2}\|g\|_{2}\|h\|_{\text {DBMO }}
\end{aligned}
$$

\section{Some estimates on singular integrals}

To begin, we have the following lemma.

Lemma 3.1. Under the assumptions of Theorem 1.4, for each $Q \in \mathbf{P}^{+}$, there exist functions $b_{Q}^{1} \in L^{p}(Q), b_{Q}^{2} \in L^{q}(Q)$ obeying the normalization

$$
\left[b_{Q}^{1}\right]_{Q}=\left[b_{Q}^{2}\right]=1
$$

and

$$
\int_{Q}\left(\left|b_{Q}^{1}\right|^{p}+\left|T b_{Q}^{1}\right|^{q^{\prime}}+\left|b_{Q}^{2}\right|^{q}+\left|T^{*} b_{Q}^{2}\right|^{p^{\prime}}\right) d \mu \leq C \mu(Q)
$$

Proof. From Theorem 1.4, we have that pseudo-accretive systems $\left\{b_{B}^{1}\right\},\left\{b_{B}^{2}\right\}$ in which each $b_{B}^{i}$ is supported in a ball $B=B\left(z_{k}^{j}, a_{0} \delta^{j}\right)$ for some $z_{k}^{j}$ and $\delta^{j}$. By Lemma 2.1, there exists $Q=Q_{k}^{j} \in \mathbf{P}^{+}$such that $B \subset Q$. Define,

$$
b_{Q}^{i}=b_{B\left(z_{k}^{j}, c_{0} \delta^{j}\right)}^{i}\left[\int b_{B\left(z_{k}^{j}, a_{0} \delta^{j}\right)}^{i} d \mu\right]^{-1}, \quad i=1,2 .
$$

Estimates (12) and (13) then follows readily.

Next, let us introduce perfect singular integral operators on spaces of homogeneous type ([1]).

Definition 3.2. A linear operator $T$ is said to be a perfect singular integral operator if it associates with a kernel $K$ that satisfies the size condition (1), and $\langle T f, g\rangle=\left\langle T^{*} f, g\right\rangle=0$ whenever $f$ is supported $Q$ with $\int_{Q} f d \mu=0$, and $\operatorname{supp} g \cap Q=\emptyset$. 
Proposition 3.3. Let $T$ be a perfect singular integral operator satisfying

$$
\|T(1)\|_{\mathrm{DBMO}},\left\|T^{*}(1)\right\|_{\mathrm{DBMO}} \lesssim 1
$$

and the weak boundedness property:

$$
\mu(Q)^{-1}\left|\left\langle T\left(\chi_{Q}\right), \chi_{Q}\right\rangle\right| \lesssim 1 \quad \text { for all } Q \in \mathbf{P}^{+} .
$$

Then $T$ extends to a bounded operator on $L^{2}(X)$.

Proof. Let $S$ denote a test function space to be any finite linear combination of the functions $\Delta_{Q} f$ for all $Q \in \mathbf{P}^{+}$and all $f \in L^{2}(X)$. To prove Proposition 3.3, we need to show that for all $f, g \in S$, we have

$$
\langle T f, g\rangle \lesssim\|f\|_{2}\|g\|_{2}
$$

Since $T$ is a perfect singular integral operator and $\int \Delta_{Q} g=0$, one has

$$
\begin{aligned}
\langle T f, g\rangle & =\sum_{P, Q}\left\langle T \Delta_{P} f, \Delta_{Q} g\right\rangle \\
& =\sum_{Q}\left\langle T \Delta_{Q} f, \Delta_{Q} g\right\rangle+\sum_{P, Q: Q \varsubsetneqq P}\left\langle T \Delta_{P} f, \Delta_{Q} g\right\rangle+\sum_{P, Q: Q \supsetneq P}\left\langle T \Delta_{P} f, \Delta_{Q} g\right\rangle \\
& =\mathrm{I}+\mathrm{II}+\mathrm{III} .
\end{aligned}
$$

For the first term, one writes

$$
\mathrm{I}=\sum_{\mathrm{Q}} \sum_{\mathrm{Q}^{\prime}, \mathrm{Q}^{\prime \prime} \in \widetilde{\mathrm{Q}}}\left([\mathrm{f}]_{\mathrm{Q}^{\prime}}-[\mathrm{f}]_{\mathrm{Q}}\right)\left([\mathrm{g}]_{\mathrm{Q}^{\prime \prime}}-[\mathrm{g}]_{\mathrm{Q}}\right)\left\langle\mathrm{T}\left(\chi_{\mathrm{Q}^{\prime}}\right), \chi_{\mathrm{Q}^{\prime \prime}}\right\rangle
$$

which, together with the property (14) and the condition (1) of $K$, gives

$$
|\mathrm{I}| \lesssim \sum_{Q}\left\|\Delta_{Q} f\right\|_{2}\left\|\Delta_{Q} g\right\|_{2} \lesssim\left\{\sum_{Q}\left\|\Delta_{Q} f\right\|_{2}^{2}\right\}^{\frac{1}{2}}\left\{\sum_{Q}\left\|\Delta_{Q} g\right\|_{2}^{2}\right\}^{\frac{1}{2}}=\|f\|_{2}\|g\|_{2} .
$$

Consider the term II. Observe that $\sum_{P: Q \nsubseteq P} \Delta_{Q} f(x)=E_{Q} f(x)$, if $x \in Q$. We apply Lemma 2.4 to obtain

$$
|\mathrm{II}| \lesssim \sum_{Q}\left|\left\langle T(1), \Delta_{Q} g\right\rangle[f]_{Q}\right| \lesssim\|f\|_{2}\|g\|_{2}\|T(1)\|_{\mathrm{DBMO}}
$$

Estimate of III is similar to that of II but with $T$ replaced by $T^{*}$. This proves (15), and then completes the proof of Proposition 3.3.

From Proposition 3.3, together with an argument as in [1, Corollary 6.3], we have the following corollary. 
Corollary 3.4. Suppose that $T$ is a perfect singular integral operator satisfying

$$
\left\|T\left(\chi_{Q}\right)\right\|_{L^{1}(Q)},\left\|T^{*}\left(\chi_{Q}\right)\right\|_{L^{1}(Q)} \lesssim \mu(Q) \quad \text { for all } Q \in \mathbf{P}^{+} .
$$

Then $T$ extends to a bounded operator on $L^{2}(X)$.

Let $\boldsymbol{P} \subseteq \boldsymbol{P}^{+}$. We say a locally integrable function $b$ is pseudo-accretive on $\boldsymbol{P}$ if $\left|[b]_{Q}\right| \gtrsim 1$ for all $Q \in \boldsymbol{P}$. In addition, if we have the property $\left|[b]_{Q^{\prime}}\right| \gtrsim 1$ for all $Q^{\prime} \in \tilde{Q}$ where $Q \in \boldsymbol{P}$, we say that $b$ is strongly pseudo-accretive on $\boldsymbol{P}$. Given such $b$, we define

$$
E_{Q}^{b}(f):=\frac{E_{Q}(f)}{E_{Q}(b)}=\frac{\int_{Q} f d \mu}{\int_{Q} b d \mu} \cdot \chi_{Q}, \quad \Delta_{Q}^{b}(f):=\sum_{Q^{\prime} \in \widetilde{Q}} E_{Q^{\prime}}^{b}(f)-E_{Q}^{b}(f) .
$$

Define $S^{b}(P)$ as a test function space to be any finite linear combination of functions $C b \chi_{P}$ and $\Delta_{Q}^{b} f$, for all $Q \in \operatorname{Tree}(P)$ and all $f \in L^{2}(P)$.

Lemma 3.5. Let $T$ be a convex tree. Let $1 \leq p \leq \infty$ with the dual exponent, i.e., $\frac{1}{p}+\frac{1}{p^{\prime}}=1$. Suppose that $b$ is strongly pseudo-accretive on $T$ and $\|b\|_{\text {mean }^{*}(T)} \lesssim 1$. Then for every $f, g \in L^{2}(X)$, we have that

$$
\sum_{Q \in T}\left\|\Delta_{Q}^{b} f\right\|_{2}^{2} \lesssim\|f\|_{2}^{2} \quad \text { and } \quad \sum_{Q \in T}\left|\left\langle f, \Delta_{Q}^{b} g\right\rangle\right| \lesssim \mu\left(Q_{T}\right)^{\frac{1}{2}}\left\||f|^{p^{\prime}}\right\|_{m_{\text {mean }}^{*}(T)}^{\frac{1}{p^{\prime}}}\|g\|_{2} .
$$

Moreover, we have

$$
\sum_{Q \in T}\left|\left\langle b^{\prime} f, \Delta_{Q}^{b} g\right\rangle\right| \lesssim \mu\left(Q_{T}\right)^{\frac{1}{2}}\left\||f|^{p^{\prime}}\right\|_{\text {mean }^{*}(T)}^{\frac{1}{p^{\prime}}}\left\|\left|b^{\prime}\right|^{p}\right\|_{\text {mean }(T)}^{\frac{1}{p}}\|g\|_{2}
$$

Proof. For the proof, we refer to [1, Lemma 6.7] for the details.

Lemma 3.6. Let $1 \leq p, q \leq \infty, Q^{\prime}, Q, P \in \boldsymbol{P}^{+}$with $Q \subseteq P$ and $Q^{\prime} \in \widetilde{Q}$. If there exists some $K \gtrsim 1$ such that $\int_{Q^{\prime}}\left(\left|T b_{P}^{1}\right|^{q^{\prime}}+\left|b_{P}^{1}\right|^{p}\right) d \mu \lesssim K \mu\left(Q^{\prime}\right)$, where $\frac{1}{q}+\frac{1}{q^{\prime}}=1$, then $\int_{Q}\left|T\left(b_{P}^{1} \chi_{Q^{\prime}}\right)\right| d \mu \lesssim K \mu\left(Q^{\prime}\right)$. Similarly for $b_{P}^{2}$ (but with $T$ replaced by $T^{*}$ ).

Proof. For the proof, we refer to [1, Corollary 6.10] for the details.

Next, we state the following lemma, which supplies an important tool in the proof of Theorem 1.4.

Proposition 3.7. Let $1<p, q \leq \infty$ with the dual exponent $p^{\prime}, q^{\prime}$. Let $P \in \mathbf{P}^{+}$. Under the assumptions of Theorem 1.4, we can partition

where

$$
\operatorname{Tree}(P)=T_{1} \cup \mathbf{P}_{\text {buffer }} \cup \bigcup_{T^{\prime} \in \boldsymbol{T}} T^{\prime},
$$


(i) $\boldsymbol{T}$ is a collection of disjoint complete trees in $\operatorname{Tree}(P)$ whose tops form a $(1-\varepsilon)$-packing of Tree $(P)$ for some $0<\varepsilon \ll 1$ (depending only on the implicit constant in (13));

(ii) $T_{1}$ is a tree with top $P$ such that $b_{P}^{1}$ is strongly pseudo-accretive on $T_{1}$ (with constant perhaps depending on $\varepsilon$ );

(iii) $\mathbf{P}_{\text {buffer }}$ is a 2-packing of Tree $(P), T_{1} \cup \mathbf{P}_{\text {buffer }}$ is convex, $b_{P}^{1}$ is pseudoaccretive on $T_{1} \cup \mathbf{P}_{\text {buffer }}$ and we have the mean bounds

$$
\left\|\left|b_{P}^{1}\right|^{p}+\left|T b_{P}^{1}\right|^{q^{\prime}}\right\|_{\text {mean* }^{*}\left(T_{1} \cup \mathbf{P}_{b u f f e r}\right)} \lesssim 1
$$

(with the implicit constant depending on $\varepsilon$ );

(iv) we have the decomposition

$$
f=[f]_{P} b_{P}^{1}+\sum_{Q \in T_{1}} \Delta_{Q}^{b_{P}^{1}} f \cdot b_{P}^{1}+\sum_{T^{\prime} \in \boldsymbol{T}}\left(f \chi_{Q_{T^{\prime}}}-[f]_{Q_{T^{\prime}}} b_{Q_{T^{\prime}}}^{1}\right)+\sum_{Q \in \mathbf{P}_{b u f f e r}} \varphi_{Q}
$$

whenever $f \in S^{b}(P)$, where the "buffer functions" $\varphi_{Q}$ are supported on $Q$, have mean zero, and take the form $\varphi_{Q}=\sum_{Q^{\prime} \in \widetilde{Q}}\left(a_{Q^{\prime}} b_{P}^{1} \chi_{Q^{\prime}}+a_{Q^{\prime}}^{\prime} b_{Q^{\prime}}^{1}\right)$, where the coefficients $a_{Q^{\prime}}$ and $a_{Q^{\prime}}^{\prime}$ depend on $f$ and the $b_{P}^{1}$ and obey the bounds

$$
\sum_{Q^{\prime} \in \widetilde{Q}}\left|a_{Q^{\prime}}\right|+\left|a_{Q^{\prime}}^{\prime}\right| \lesssim\|f\|_{\infty}
$$

A similar statement holds with $b_{P}^{1}$ and $T b_{P}^{1}$ replaced by $b_{P}^{2}$ and $T^{*} b_{P}^{2}$ (but the sets $T_{1}, \boldsymbol{P}_{\text {buffer }}$ and $\boldsymbol{T}$ are different then).

Proof. The proof can be obtained by making minor modifications with [1, Lemma 6.11]. We omit the details here.

\section{Proof of Theorem 1.4}

The aim of this section is to prove our main result, Theorem 1.4. For clarity, we divide it into two steps.

Step I: $T$ is a perfect singular operator. Let A be the smallest constant such that $\left\|T^{*} \chi_{P}\right\|_{L^{1}(P)} \leq A \mu(P)$ for all $P \in \mathbf{P}^{+}$. The assumptions on $T$ imply $A$ is finite. We claim that the bound $A=O(1)$, independent of truncations of the standard kernel $K$. If the claim holds, then Theorem 1.4 follows from Corollary 3.4.

Let us prove our claim. Indeed, we shall show that

$$
\left|\int_{P} T f d \mu\right| \leq((1-\varepsilon) A+O(1)) \mu(P)\|f\|_{\infty}
$$


for all $P \in \mathbf{P}^{+}$and $f \in S^{b}(P)$, and some $0<\varepsilon<1$ depending only on the implicit constant in (13). By duality this implies that $A \leq(1-\varepsilon) A+O(1)$, which proves the desired bound on $A$. Apply the equality (17) of Proposition 3.7 (with the $b_{P}^{1}$ ), we estimate

$$
\begin{aligned}
\left|\int_{P} T f d \mu\right| \leq & \left|\int_{P}[f]_{P} T b_{P}^{1} d \mu\right|+\left|\sum_{Q \in T_{1}} \int_{P} T\left(b_{P}^{1} \Delta_{Q}^{b_{P}^{1}} f\right) d \mu\right| \\
& +\sum_{T^{\prime} \in \mathbf{T}}\left|\int_{P} T\left(f \chi_{Q_{T^{\prime}}}-[f]_{Q_{T^{\prime}}} b_{Q_{T^{\prime}}}^{1}\right) d \mu\right|+\sum_{Q \in \mathbf{P}_{b u f f e r}}\left|\int_{P} T \varphi_{Q} d \mu\right| \\
= & : \mathrm{I}+\mathrm{II}+\mathrm{III}+\mathrm{IV} .
\end{aligned}
$$

Observe that $\mathrm{I} \leq O(1)\|f\|_{\infty} \mu(P)$, by Hölder's inequality and the condition (13). For term III, we use the fact that the function $f \chi_{Q_{T^{\prime}}}-[f]_{Q_{T^{\prime}}} b_{Q_{T^{\prime}}}^{1}$ has mean zero on $Q_{T^{\prime}}$ to show that

$$
\begin{aligned}
\mathrm{III} & \leq \sum_{T^{\prime} \in \mathbf{T}}\left\{\left|\left\langle f \chi_{Q_{T^{\prime}}}, T^{*} \chi_{Q_{T^{\prime}}}\right\rangle\right|+\left|[f]_{Q_{T^{\prime}}} \int_{Q_{T^{\prime}}} T b_{Q_{T^{\prime}}}^{1} d \mu\right|\right\} \\
& \leq \sum_{T^{\prime} \in \mathbf{T}}\left\{A\|f\|_{\infty} \mu\left(Q_{T^{\prime}}\right)+O(1)\|f\|_{\infty} \mu\left(Q_{T^{\prime}}\right)\right\} \\
& \leq((1-\varepsilon) A+O(1))\|f\|_{\infty} \mu(P),
\end{aligned}
$$

where the last inequality is obtained by using the fact that $\sum_{T^{\prime} \in \mathbf{T}} \mu\left(Q_{T^{\prime}}\right) \leq$ $(1-\varepsilon) \mu(P)$.

Note that $\mathbf{P}_{\text {buffer }}$ is a 2-packing. We use Lemma 3.6, the inequality (16) and the bounds (18) to obtain

$$
\mathrm{IV} \leq O(1)\left(\sum_{Q \in \mathbf{P}_{\text {buffer }}}\|f\|_{\infty} \mu(Q)\right) \leq O(1)\|f\|_{\infty} \mu(P) .
$$

The left of the proof is to estimate the second term II. Define

$$
B:=\left.\sup _{\|\left. f\right|_{\infty} \leq 1}\left\|\left\langle T^{*} \chi_{P}, b_{P}^{1} \Delta_{Q}^{b_{P}^{1}} f\right\rangle\right\|\right|_{\operatorname{size}^{*}\left(T_{1}\right)} .
$$

We need to show that

$$
\sum_{Q \in T_{1} \cap \operatorname{Tree}\left(P^{\prime}\right)}\left|\left\langle T^{*} \chi_{P}, b_{P}^{1} \Delta_{Q}^{b_{P}^{1}} f\right\rangle\right| \leq((1-\varepsilon) B+O(1)) \mu\left(P^{\prime}\right)
$$

for all $P^{\prime} \subseteq P$ and $\|f\|_{\infty} \leq 1$, and this gives that $B=O(1)$. 
Let us prove (20). Fix a $P^{\prime}$. By Proposition 3.7 (with the $b_{P^{\prime}}^{2}$ ), we have $\operatorname{Tree}\left(P^{\prime}\right)=T_{2} \cup \mathbf{P}_{\text {buffer }}^{\prime} \cup \bigcup_{T^{\prime} \in \mathbf{T}^{\prime}} T^{\prime}$. Putting it into (20), we have

$$
\begin{aligned}
\sum_{Q \in T_{1} \cap \operatorname{Tree}\left(P^{\prime}\right)} & \left|\left\langle T^{*} \chi_{P}, b_{P}^{1} \Delta_{Q}^{b_{P}^{1}} f\right\rangle\right| \\
& \leq\left(\sum_{Q \in T_{1} \cap \underbrace{\cup}_{T^{\prime} \in \mathbf{T}^{\prime}} T^{\prime}}+\sum_{Q \in T_{1} \cap \mathbf{P}_{b u f f e r}^{\prime}}+\sum_{Q \in T_{1} \cap T_{2}}\right)\left|\left\langle T^{*} \chi_{P}, b_{P}^{1} \Delta_{Q}^{b_{P}^{1}} f\right\rangle\right| \\
& =: \mathrm{II}_{1}+\mathrm{II}_{2}+\mathrm{II}_{3} .
\end{aligned}
$$

Since $\mathbf{T}^{\prime}$ is an $(1-\varepsilon)$-packing, $\mathrm{II}_{1} \leq(1-\varepsilon) B \mu\left(P^{\prime}\right)$. For any $Q \in T_{1} \cap \mathbf{P}_{\text {buffer }}^{\prime}$, it follows from by the Lemma 3.6 that

$$
\left|\left\langle T^{*} \chi_{P}, b_{P}^{1} \Delta_{Q}^{b_{P}^{1}} f\right\rangle\right|=\left|\int_{P} T\left(b_{P}^{1} \Delta_{Q}^{b_{P}^{1}} f\right) d \mu\right|=\left|\int_{Q} T\left(b_{P}^{1} \Delta_{Q}^{b_{P}^{1}} f\right) d \mu\right| \leq O(1) \mu(Q),
$$

and hence, $\mathrm{II}_{2} \leq O(1) \mu\left(P^{\prime}\right)$.

In order to estimate $\mathrm{II}_{3}$, we fix $Q \in T_{1} \cap T_{2}$, and write $F=\chi_{Q}-\frac{b_{P^{\prime}}^{2} \chi_{Q}}{\left[b_{P^{\prime}}^{2}\right]_{Q}}$. Using Lemma 3.5, we can estimate

$$
\left|\left\langle T^{*} \chi_{P}, b_{P}^{1} \Delta_{Q}^{b_{P}^{1}} f\right\rangle\right| \leq\left|\left\langle T^{*} \frac{b_{P^{\prime}}^{2}}{\left[b_{P^{\prime}}^{2}\right]_{Q}}, b_{P}^{1} \Delta_{Q}^{b_{P}^{1}} f\right\rangle\right|+\left|\left\langle T^{*} F, b_{P}^{1} \Delta_{Q}^{b_{P}^{1}} f\right\rangle\right| .
$$

For the first term is $\left|O(1) \mu\left(P^{\prime}\right)\right|$ by the pseudo-accretivity of $b_{P^{\prime}}^{2}$. For the second term, it follows from an argument as in $[1$, p. 47] that it suffices to control the commutator $\left|\left\langle T^{*} F, b_{P}^{1} \Delta_{Q}^{b_{P}^{1}} f\right\rangle-\left\langle T^{*}\left(F \Delta_{Q}^{b_{P}^{1}} f\right), b_{P}^{1}\right\rangle\right|$. If $F$ has mean zero on all $Q^{\prime} \in \widetilde{Q}$, then this commutator would be zero. Hence, we may freely replace $F$ by $\sum_{Q^{\prime} \in \widetilde{Q}}[F]_{Q^{\prime}} \chi_{Q^{\prime}}$. If we throw the $T^{*}$ onto the other side and apply the Lemma 3.6, this commutator is bounded by $O(1) \mu(Q)^{\frac{1}{2}}\left\|\Delta_{Q}^{b_{P}^{1}} f\right\|_{2} \sum_{Q^{\prime} \in \widetilde{Q}}\left|[F]_{Q^{\prime}}\right|$. A direct computation shows that

$$
\sum_{Q^{\prime} \in \widetilde{Q}}\left|[F]_{Q^{\prime}}\right|=\sum_{Q^{\prime} \in \widetilde{Q}}\left|1-\frac{\left[b_{P^{\prime}}^{2}\right]_{Q^{\prime}}}{\left[b_{P^{\prime}}^{2}\right]_{Q}}\right| \leq \sum_{Q^{\prime} \in \widetilde{Q}}\left|\left[b_{P^{\prime}}^{2}\right]_{Q^{\prime}}-\left[b_{P^{\prime}}^{2}\right]_{Q}\right| \lesssim\left\|\Delta_{Q} b_{P^{\prime}}^{2}\right\|_{2} \mu(Q)^{-\frac{1}{2}},
$$

and then $\mathrm{II}_{3} \leq O(1) \mu\left(P^{\prime}\right)$ follows by the pseudo-accretivity of $b_{P^{\prime}}^{2}$. This proves (20), and then completes the proof of Theorem 1.4 for perfect singular operators.

Step II: A complete proof of Theorem 1.4. The proof heavily depends on the BCR algorithm for singular integrals on spaces of homogeneous type, whose idea is borrowed from [6]. For every $f, g \in S$, define

$$
\begin{aligned}
& a(j, k, l, f):=\mu\left(Q_{l}^{j}\right)^{-\frac{1}{2}} \int_{Q_{k}^{j}} \int_{Q_{l}^{j}} K(x, y)\left(\frac{\Delta_{Q_{l}^{j}} f(y)}{\left\|\Delta_{Q_{l}^{j}} f(y)\right\|_{2}}\right) d \mu(y) d \mu(x) \\
& b(j, k, l, g):=\mu\left(Q_{l}^{j}\right)^{-\frac{1}{2}} \int_{Q_{k}^{j}} \int_{Q_{l}^{j}} K(y, x)\left(\frac{\Delta_{Q_{l}^{j}} g(y)}{\left\|\Delta_{Q_{l}^{j}} g(y)\right\|_{2}}\right) d \mu(y) d \mu(x) .
\end{aligned}
$$


It follows from Lemma 2.1 that

$$
\begin{aligned}
\langle g, T f\rangle & =\sum_{j \in \mathbb{Z}}\left(\left\langle g, E_{j} T \Delta_{j} f\right\rangle+\left\langle E_{j} T^{*} \Delta_{j} g, f\right\rangle+\left\langle g, \Delta_{j} T \Delta_{j} f\right\rangle\right) \\
& =:\langle g, U f\rangle+\langle V g, f\rangle+\langle g, W f\rangle
\end{aligned}
$$

where

$$
\begin{aligned}
U f & =\sum_{j, k, l} \mu\left(Q_{l}^{j}\right)^{\frac{1}{2}} \mu\left(Q_{k}^{j}\right)^{-1} a(j, k, l, f)\left\|\Delta_{Q_{l}^{j}} f\right\|_{2} \chi_{Q_{k}^{j}} \\
V g & =\sum_{j, k, l} \mu\left(Q_{l}^{j}\right)^{\frac{1}{2}} \mu\left(Q_{k}^{j}\right)^{-1} b(j, k, l, g)\left\|\Delta_{Q_{l}^{j}} g\right\|_{2} \chi_{Q_{k}^{j}} \\
W f & =\sum_{j, k, l: k \neq l} \Delta_{Q_{k}^{j}} T \Delta_{Q_{l}^{j}} f .
\end{aligned}
$$

Let us go further and modify formally $U, V, W$. Set

$$
\begin{aligned}
& a^{\prime}(j, k, l, f)= \begin{cases}a(j, k, l, f) & \text { if } k \neq l \\
-\sum_{k^{\prime}: k^{\prime} \neq k} a\left(j, k^{\prime}, k, f\right) & \text { if } k=l\end{cases} \\
& b^{\prime}(j, k, l, f)= \begin{cases}b(j, k, l, f) & \text { if } k \neq l \\
-\sum_{k^{\prime}: k^{\prime} \neq k} b\left(j, k, k^{\prime}, f\right) & \text { if } k=l .\end{cases}
\end{aligned}
$$

One writes $W^{\prime} f=W f$. Define

$$
\begin{aligned}
U^{\prime} f & =\sum_{j, k, l} \mu\left(Q_{l}^{j}\right)^{\frac{1}{2}} \mu\left(Q_{k}^{j}\right)^{-1} a^{\prime}(j, k, l, f)\left\|\Delta_{Q_{l}^{j}} f\right\|_{2} \chi_{Q_{k}^{j}} \\
V^{\prime} g & =\sum_{j, k, l} \mu\left(Q_{l}^{j}\right)^{\frac{1}{2}} \mu\left(Q_{k}^{j}\right)^{-1} b^{\prime}(j, k, l, g)\left\|\Delta_{Q_{l}^{j}} g\right\|_{2} \chi_{Q_{k}^{j}} .
\end{aligned}
$$

Then the following result holds.

Proposition 4.1. The operator $T^{\prime}=U^{\prime}+\left(V^{\prime}\right)^{*}+W^{\prime}$ is bounded on $L^{2}(X)$. Moreover, $T-T^{\prime}$ is a perfect singular operator.

Proof. We first prove $L^{2}$-boundedness of $U^{\prime}$. For every $R \in \mathbb{N}$, we define

$$
a_{R}^{\prime}(j, k, l, f)= \begin{cases}a^{\prime}(j, k, l, f), & \text { if } C_{0} \leq \frac{\rho\left(z_{k}^{j}, z_{l}^{j}\right)}{\delta^{j+R}} \leq C_{0} \delta \\ -\sum_{k^{\prime}: C_{0} \leq \frac{\rho\left(z_{k^{\prime}}^{j}, z_{l}^{j}\right)}{\delta^{j}+R} \leq C_{0} \delta} a^{\prime}\left(j, k^{\prime}, l, f\right), & \text { if } k=l \\ 0, & \text { otherwise }\end{cases}
$$


and $U_{R}^{\prime} f=\sum_{j, k, l} \mu\left(Q_{l}^{j}\right)^{\frac{1}{2}} \mu\left(Q_{k}^{j}\right)^{-1} a_{R}^{\prime}(j, k, l, f)\left\|\Delta_{Q_{l}^{j}} f\right\|_{2} \chi_{Q_{k}^{j}}$. Since $\sum_{k} a_{R}^{\prime}(j, k, l, f)$ $=0$, we have that $U^{\prime} f=\sum_{R=0}^{\infty} U_{R}^{\prime} f$. In order to prove $L^{2}$-boundedness of $U^{\prime}$, it suffices to show that

$$
\left\|U_{R}^{\prime} f\right\|_{2} \lesssim R^{\frac{1}{2}} \delta^{-R \varepsilon}\|f\|_{2}, \quad \forall R \in \mathbb{N} .
$$

Let us prove (21). Using the conditions (1) and (2) of the kernel $K$, together with the fact that $\int \Delta_{Q_{l}^{j}} f=0$, we can estimate

$$
\sum_{k}\left|a_{R}^{\prime}(j, k, l, f)\right| \lesssim \delta^{-R \varepsilon}
$$

and

$$
\sum_{l} \mu\left(Q_{l}^{j}\right) \mu\left(Q_{k}^{j}\right)^{-1}\left|a_{R}^{\prime}(j, k, l, f)\right| \lesssim \delta^{-R \varepsilon}
$$

Set $f_{j}=\Delta_{j} f$. Using estimates (22) and (23), we obtain

$$
\begin{aligned}
\left\|U_{R}^{\prime} f_{j}\right\|_{2}^{2} & \leq \sum_{k}\left(\sum_{l} \mu\left(Q_{l}^{j}\right) \mu\left(Q_{k}^{j}\right)^{-1}\left|a_{R}^{\prime}(j, k, l, f)\right|\right)\left(\sum_{l}\left|a_{R}^{\prime}(j, k, l, f)\right| \cdot\left\|\Delta_{Q_{l}^{j}} f\right\|_{2}^{2}\right) \\
& \lesssim \delta^{-2 R \varepsilon}\left\|f_{j}\right\|_{2}^{2} .
\end{aligned}
$$

One writes

$$
\begin{aligned}
\left\|U_{R}^{\prime} f\right\|_{2}^{2} & =\sum_{j, j^{\prime}}\left\langle U_{R}^{\prime} f_{j}, \overline{U_{R}^{\prime} f_{j^{\prime}}}\right\rangle \\
& =\left(\sum_{\left|j-j^{\prime}\right| \leq R+C_{3}}+\sum_{j>j^{\prime}+R+C_{3}}+\sum_{j^{\prime}>j+R+C_{3}}\right)\left\langle U_{R}^{\prime} f_{j}, \overline{U_{R}^{\prime} f_{j^{\prime}}}\right\rangle \\
& =\mathrm{I}+\mathrm{II}+\mathrm{III},
\end{aligned}
$$

where $C_{3}$ is a constant to be chosen later.

The first term can be computed as follows.

$$
\begin{aligned}
|\mathrm{I}| \leq \sum_{\left|j-j^{\prime}\right| \leq R+C_{3}}\left\|U_{R}^{\prime} f_{j}\right\|_{2}\left\|U_{R}^{\prime} f_{j^{\prime}}\right\|_{2} & \lesssim \sum_{\left|j-j^{\prime}\right| \leq R+C_{3}}\left(\left\|U_{R}^{\prime} f_{j}\right\|_{2}^{2}+\left\|U_{R}^{\prime} f_{j^{\prime}}\right\|_{2}^{2}\right) \\
& \lesssim R \delta^{-2 R \varepsilon}\|f\|_{2}^{2} .
\end{aligned}
$$

Consider the second term. It can be seen that it is less than

$$
\sum_{\substack{k, l, j \\ k^{\prime}, l^{\prime}, j^{\prime}}} \sum_{\substack{j>j^{\prime}+R+C_{3} \\ Q_{k^{\prime}}^{j^{\prime}} \subseteq Q_{k}^{j}}} \frac{\left[\mu\left(Q_{l}^{j}\right) \mu\left(Q_{l^{\prime}}^{j^{\prime}}\right)\right]^{\frac{1}{2}}}{\mu\left(Q_{k}^{j}\right)} \mid a_{R}^{\prime}(j, k, l, f)\left\|a_{R}^{\prime}\left(j^{\prime}, k^{\prime}, l^{\prime}, f\right)\right\| \Delta_{Q_{l}^{j}} f\left\|_{2}\right\| \Delta_{Q_{l^{\prime}}^{j^{\prime}}} f \|_{2} .
$$


Noting that $\operatorname{supp} a_{R}^{\prime}\left(j^{\prime}, k^{\prime}, l^{\prime}, f\right) \subset\left\{\rho\left(z_{k^{\prime}}^{j^{\prime}}, z_{l^{\prime}}^{j^{\prime}}\right) \leq C_{0} \delta^{j^{\prime}+R}\right\}$ and $Q_{k^{\prime}}^{j^{\prime}} \subseteq Q_{k}^{j}$, we have that $Q_{l^{\prime}}^{j^{\prime}} \subseteq\left\{x \mid \rho\left(x, Q_{k}^{j}\right)<C_{4} \delta^{j^{\prime}+R}\right\}$, where $C_{4}=(A+1)^{2}\left(C_{0}+C_{1}\right)$. Set $H_{k}^{j}=\left\{x \mid \in X \backslash Q_{k}^{j}, \rho\left(x, Q_{k}^{j}\right)<C_{4} \delta^{j^{\prime}+R}\right\} \cup\left\{x \mid \in Q_{k}^{j}, \rho\left(x, X \backslash Q_{k}^{j}\right)<C_{4} \delta^{j^{\prime}+R}\right\}$. If $Q_{l^{\prime}}^{j^{\prime}} \subseteq Q_{k}^{j}$, and $\rho\left(z_{l^{\prime}}^{j^{\prime}}, X \backslash Q_{k}^{j}\right)>C_{4} \delta^{j^{\prime}+R}$, then $Q_{l^{\prime}}^{j^{\prime}} \subset H_{k}^{j}$. This yields that $\mu\left(H_{k}^{j}\right) \lesssim \delta^{\left(j^{\prime}+R-j\right) \eta} \mu\left(Q_{k}^{j}\right)$. We have

$$
\begin{aligned}
|\mathrm{II}| & \lesssim \delta^{-R \varepsilon} \sum_{j, j^{\prime}, k, l} \sum_{l^{\prime}: Q_{l^{\prime}}^{j^{\prime}} \subset H_{k}^{j}} \frac{\left[\mu\left(Q_{l}^{j}\right) \mu\left(Q_{l^{\prime}}^{j^{\prime}}\right)\right]^{\frac{1}{2}}}{\mu\left(Q_{k}^{j}\right)}\left|a_{R}^{\prime}(j, k, l, f)\right|\left\|\Delta_{Q_{l}^{j}} f\right\|_{2}\left\|\Delta_{Q_{l^{\prime}}^{j^{\prime}}} f\right\|_{2} \\
& \lesssim \delta^{-\frac{3 R \varepsilon}{2}} \sum_{j, j^{\prime}}\left\{\sum_{\substack{k, l, l^{\prime}: \\
Q_{l^{\prime}}^{j^{\prime}} \in H_{k}^{j}}} \mid a_{R}^{\prime}(j, k, l, f)\left\|\Delta_{Q_{l}^{j}} f\right\|_{2}^{2}\left(\frac{\mu\left(Q_{l^{\prime}}^{j^{\prime}}\right)}{\mu\left(Q_{k}^{j}\right)}\right)\right\}^{\frac{1}{2}}\left\{\sum_{\substack{k, l^{\prime}: \\
Q_{l^{\prime}} j^{j}}}\left\|\Delta_{Q_{k}^{j^{\prime}}} f\right\|_{2}^{2}\right\}^{\frac{1}{2}} \\
& \lesssim \delta^{-2 R \varepsilon}\|f\|_{2}^{2} .
\end{aligned}
$$

The same argument shows that $|\mathrm{III}| \lesssim \delta^{-2 R \varepsilon}\|f\|_{2}^{2}$. This proves estimate (21), and then gives the $L^{2}$-boundedness of $U^{\prime}$.

Similarly, $V^{\prime}$ is bounded on $L^{2}(X)$. For the operator $W^{\prime}$, we use the conditions (1) and (2) of the kernel $K$ to estimate

$$
\left|\left\langle g, W^{\prime} f\right\rangle\right| \leq \sum_{j, k, l: k \neq l}\left|\left\langle\Delta_{Q_{k}^{j}} g, \Delta_{Q_{l}^{j}} T f\right\rangle\right| \lesssim \sum_{j}\left\|f_{j}\right\|_{2}\left\|g_{j}\right\|_{2} \lesssim\|f\|_{2}\|g\|_{2},
$$

which gives $L^{2}$-boundedness of $W^{\prime}$. Hence, $T^{\prime}$ is bounded on $L^{2}(X)$.

Finally, let us verify that $T-T^{\prime}$ is a perfect singular operator. Note that the condition (1) for $T-T^{\prime}$ is satisfied. Now, if $\operatorname{supp} f \subset Q_{k^{\prime}}^{j^{\prime}}, \int f=0$ and $\operatorname{supp} g \subset\left(Q_{k^{\prime}}^{j^{\prime}}\right)^{c}$, we need to show that

$$
\left\langle g,\left(T-T^{\prime}\right) f\right\rangle=0 .
$$

Observe that $\left\langle g,\left(U-U^{\prime}\right) f\right\rangle=\sum_{k} \int_{X} T\left(\Delta_{Q_{k}^{j}} f\right) d \mu E_{Q_{k}^{j}} g$. Hence, $\Delta_{Q_{k}^{j}} f=0$ unless $Q_{k}^{j} \subseteq Q_{k^{\prime}}^{j^{\prime}}$, and this leads to $E_{Q_{k}^{j}} g=0$. The similar argument shows that $\left\langle g,\left(V^{*}-\left(V^{\prime}\right)^{*}\right) f\right\rangle=\left\langle g,\left(W-W^{\prime}\right) f\right\rangle=0$. This proves $(24)$, and then $T-T^{\prime}$ is a perfect singular operator. The proof of Proposition 4.1 is complete.

Proof of Theorem 1.4. From Proposition 4.1 and a standard argument( [9]), it follows that the operator $T^{\prime}$ is bounded on $L^{p}$ for all $1<p<\infty$. Note that $q^{\prime} \leq p$. We have

$$
\begin{aligned}
& \left(\frac{1}{\mu(Q)} \int_{Q}\left|\left(T-T^{\prime}\right) b_{Q}^{1}\right|^{q^{\prime}} d \mu\right)^{\frac{1}{q^{\prime}}} \\
& \lesssim\left(\frac{1}{\mu(Q)} \int_{Q}\left|T b_{Q}^{1}\right|^{q^{\prime}} d \mu\right)^{\frac{1}{q^{\prime}}}+\left(\frac{1}{\mu(Q)} \int_{Q}\left|T^{\prime} b_{Q}^{1}\right|^{p} d \mu\right)^{\frac{1}{p}} \lesssim 1
\end{aligned}
$$


and

$$
\left(\frac{1}{\mu(Q)} \int_{Q}\left|\left(T-T^{\prime}\right) b_{Q}^{2}\right|^{p^{\prime}} d \mu\right)^{\frac{1}{p^{\prime}}} \lesssim 1 .
$$

From Step I, we have that $T-T^{\prime}$ is bounded on $L^{2}(X)$. This completes of the proof of Theorem 1.4.

Acknowledgements. The authors would like to thank the referee for helpful comments, and thank Y. S. Han for helpful discussions and suggestions. C. Tan was supported by Youth Research Fund of Shantou University. L. Yan was supported by NCET of Ministry of Education of China and NNSF of China (Grant No. 10771221).

\section{References}

[1] Auscher, P., Hofmann, S., Muscalu, C., Tao, T. and Thiele, C., Carleson measures, trees, extrapolation, and T(b) theorems. Publ. Mat. 46 (2002), 257 - 325.

[2] Auscher, P. and Yang, Q. X., BCR algorithm and the T(b) theorem. Publ. Mat. 53 (2009), 179 - 196.

[3] Beylkin, G., Coifman, R. and Rokhlin, V., Fast wavelet transforms and numerical algorithms. I. Comm. Pure Appl. Math. 44 (1991), 141 - 183.

[4] Christ, M., A $T(b)$ theorem with remarks on analytic capacity and the Cauchy integral. Colloq. Math. 60/61 (1990), $601-628$.

[5] Coifman R. and Weiss, G., Analyse Harmonique Non-Commutative sur Certains Espaces Homogenes. Lect. Notes Math. 242. Berlin: Springer 1971.

[6] Deng, D. G., Han, Y. S. and Lin, C. C., Blocking analysis on spaces of homogeneous type and the T(1) theorem. Unpublished preprint 2002.

[7] David, G., Journé, J. and Semmes, S., Opérateurs de Calderón-Zygmund, fonctions para-accrétives et interpolation (in French). Rev. Mat. Iberoamericana 1 (1985), $1-56$.

[8] Deng, D. G., Yan, L. X. and Yang, Q. X., Blocking analysis and T(1) theorem. Sci. China Ser. A 41 (1998), $801-808$.

[9] Meyer, Y., Ondelettes et Opérateurs II. Opérateurs de Calderón-Zygmund. Paris: Hermann 1990.

[10] Yang, Q. X., Fast algorithm for Calderón-Zygmund singular integral operators. Appl. Comput. Harmon. Anal. 3 (1996), $120-126$.

Received September 29, 2007 